\title{
Autoimmune pancreatitis type 1
}

INSERM

\section{Source}

INSERM. (1999). Orphanet: an online rare disease and orphan drug data base.

Autoimmune pancreatitis type 1. ORPHA:280302

Type 1 autoimmune pancreatitis is a form of autoimmune pancreatitis seen in elderly males (>60 years) and presenting with abdominal pain, steatorrhea, obstructive jaundice and other org an (bile duct, kidneys and retroperitoneum) involvement. It is thoug ht to be due to an immunoglobulin G4 (IgG4)-associated systemic disease. 